\title{
Cellular senescence in brain aging and neurodegenerative diseases: evidence and perspectives
}

\author{
Darren J. Baker ${ }^{1,2}$ and Ronald C. Petersen ${ }^{3}$ \\ Department of Biochemistry and Molecular Biology, ${ }^{1}$ Department of Pediatric and Adolescent Medicine, and ${ }^{3}$ Department of Neurology, Mayo Clinic, Rochester, Minnesota, USA
}

\begin{abstract}
Along with a general decline in overall health, most chronic degenerative human diseases are inherently associated with increasing age. Age-associated cognitive impairments and neurodegenerative diseases, such as Parkinson's and Alzheimer's diseases, are potentially debilitating conditions that lack viable options for treatment, resulting in a tremendous economic and societal cost. Most high-profile clinical trials for neurodegenerative diseases have led to inefficacious results, suggesting that novel approaches to treating these pathologies are needed. Numerous recent studies have demonstrated that senescent cells, which are characterized by sustained cell cycle arrest and production of a distinct senescence-associated secretory phenotype, accumulate with age and at sites of age-related diseases throughout the body, where they actively promote tissue deterioration. Cells with features of senescence have been detected in the context of brain aging and neurodegenerative disease, suggesting that they may also promote dysfunction. Here, we discuss the evidence implicating senescent cells in neurodegenerative diseases, the mechanistic contribution of these cells that may actively drive neurodegeneration, and how these cells or their effects may be targeted therapeutically.
\end{abstract}

\section{Introduction}

The risk of developing many pathological conditions, including neurodegenerative diseases, increases dramatically with age. Increased life expectancies due to advancements in medicine have greatly expanded the number of elderly individuals who will suffer from geriatric neurodegenerative diseases, including Parkinson's disease (PD) and Alzheimer's disease (AD). The financial cost of providing long-term care for current and future sufferers of these conditions is overwhelming. For example, in 2010, approximately 4.7 million Americans were living with $\mathrm{AD}$, the most common neurodegenerative disease; by 2050 it is projected to be around 16 million (1). Medical expenses to treat these patients, most over the age of 65 (2), exceed those to treat individuals with cancer and cardiovascular disease combined, placing an immense burden on Medicare and Medicaid (3). Unfortunately, of the numerous clinical trials to treat $\mathrm{AD}$, none have been able to demonstrate beneficial impact for patients.

As age is inherently linked with an increased predisposition to many diseases and death, people have been searching for ways to delay the rate of aging for centuries. One of the best-known attempts to reverse the process of aging has been the search for the legendary Fountain of Youth, perhaps most notoriously attributed to the explorations of Ponce de León in the 16th century. It was said that bathing

Conflict of interest: R.C. Petersen is a consultant for Biogen, Genentech, Merck, and Roche Inc. D.J. Baker holds patents licensed to or filed by Unity Biotechnology, a company developing senolytic medicines, including small molecules that selectively eliminate senescent cells (15/455,630 and 62/232,344). Research in D.J. Baker's laboratory has been reviewed by the Mayo Clinic Conflict of Interest Review Board and is being conducted in compliance with Mayo Clinic conflict of interest policies.

Reference information: / Clin Invest. 2018;128(4):1208-1216.

https://doi.org/10.1172/JCI95145. in its waters would restore the vitality of youthfulness. Unfortunately, this legend remains just that, and reversal of the aging process resides in the territory of fairy tales. The search for means to delay aging has not ceased, though, and is now being performed by biologists, not geographers and adventurers. These researchers have been working to understand the mechanisms that underlie the aging process such that we may be able to delay aging or potentially reverse it. Recent work has demonstrated that senescent cells, characterized by an arrested cell cycle that is triggered by a variety of stresses, accumulate in various tissues of age and disease (4-8), suggesting that they may actively contribute to disease pathology. Unlike a normally programmed terminal differentiation process, senescence is a distinct proinflammatory fate in which cells acquire a distinctive secretome of cytokines, chemokines, proteases, and growth factors collectively known as the senescence-associated secretory phenotype (SASP) (9). While senescent cells in peripheral tissues have been the focus of numerous recent studies, their involvement in or contribution to cognitive decline with aging or diseases of the central nervous system remains relatively unknown. In this Review, we will discuss what constitutes senescence, the evidence implicating senescence in dysfunction of the CNS, and how senescent cells may be targeted for the development of novel therapeutics to treat pathologies associated with brain aging.

\section{Properties of senescent cells}

The first experimental evidence for cellular aging in vitro came from studies conducted more than 50 years ago. In a landmark study, Leonard Hayflick and Paul Moorhead demonstrated that normal diploid human fibroblasts exhibit a limited potential for replication before entering into a state termed replicative senescence (10). Using these observations, Hayflick hypothesized that these nondividing cells contributed to aging because they had lost the ability to 
A
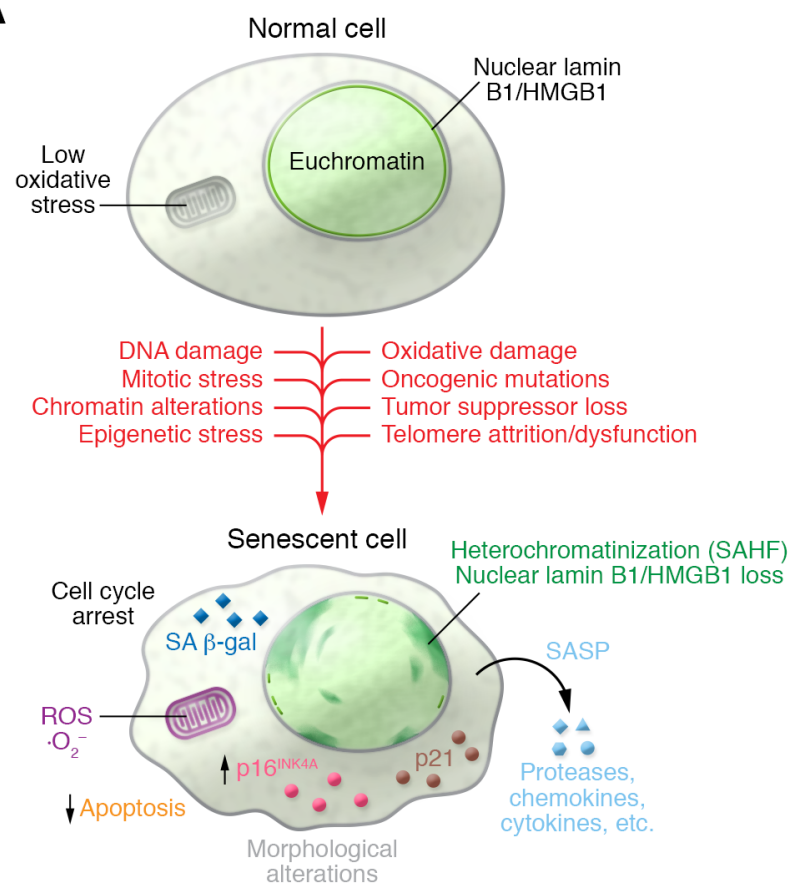

B

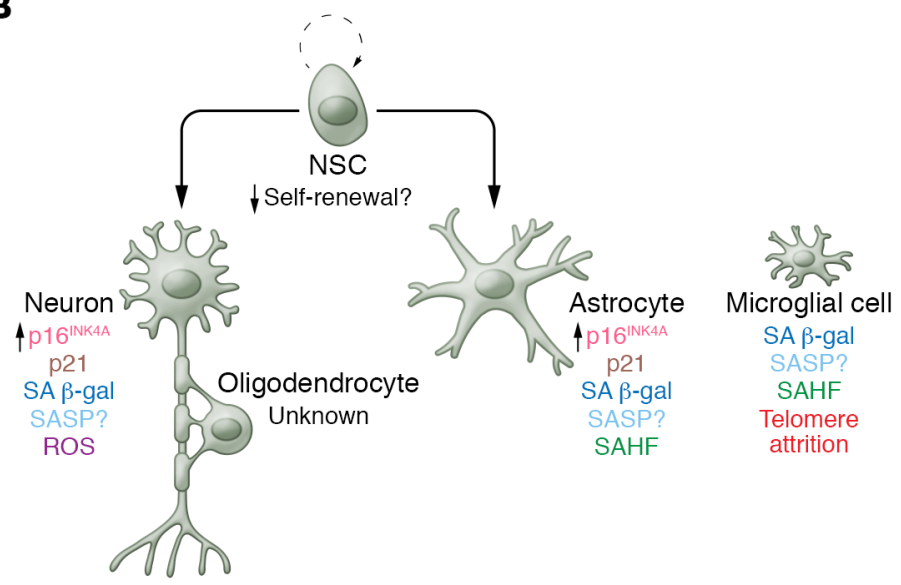

Figure 1. Hallmarks of senescence in CNS cells. (A) A number of stressors, both intrinsic and extrinsic, can stimulate the conversion of otherwise normal cells into senescent cells. Once they have entered into this state, a number of distinguishing qualities can be observed. depending on the cell type and senescence-inducing stimuli. (B) Senescent cell evidence and identity are observed in neurological aging and pathology. Commonly observed features reminiscent of senescence are indicated for the various cell types. See text for extended discussion and references. SAHF, senescence-associated heterochromatin foci; NSC, neural stem cell.

participate in repair and regeneration processes within tissues (11). This hypothesis remained untested for decades, as the molecular determinants of senescence and the ability to identify and manipulate senescent cells in vivo were then unknown. However, in the last 20 years, we have greatly advanced our understanding of the mechanisms that drive cells into senescence and the subsequent changes in tissue health that result from this process.

Attrition of telomeres, the repeating nucleotide sequences of TTAGGG located at the ends of chromosomes, was later identified as the molecular determinant underlying replicative senescence (12). During the process of DNA replication, DNA polymerases are unable to completely replicate telomeres, resulting in progressive loss with each cell division (13). Critically shortened telomeres can lead to chromosomal instability and tumor formation (14). On the other hand, cells that have become senescent permanently arrest their cell cycle and represent a robust anticancer mechanism (15). Various other stresses, including DNA damage, reactive oxygen species (ROS), strong mitogenic/oncogenic signaling, loss of certain tumor suppressors, mitotic stress, stalled DNA replication, and chromatin disruption (16-22), are also capable of inducing senescence (Figure 1).

Regardless of the initiating events, the senescent growth arrest requires a coordinated action of the $\mathrm{p} 53 / \mathrm{p} 21$ and $\mathrm{p} 16^{\mathrm{INK} 4 \mathrm{~A}} /$ retinoblastoma (RB) tumor-suppressive pathways (7). Uncapped telomeres and DNA double-strand breaks activate a DNA damage response that leads to stabilization of $\mathrm{p} 53$ through posttranslational phosphorylation by ATM and ATR serine/threonine protein kinases or by blocking of p53 degradation through p14 ${ }^{\mathrm{ARF}}$-mediated inhibition (in

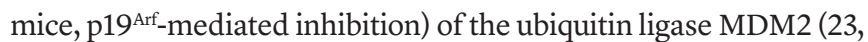
24). Transcription of the cyclin-dependent kinase inhibitor (CDKi) p21 occurs upon p53 stabilization, leading to an initial arrest of the cell cycle (25). After this initial transient arrest, permanent arrest is controlled by p $16^{\mathrm{INK} 4 \mathrm{~A}}$ transcriptional upregulation through $\mathrm{p} 38$ (26) and/or ERK signaling (27). Once present, p16 $6^{\mathrm{INK} 4 \mathrm{~A}}$ inhibits the activity of both CDK4 and CDK6, thereby leading to RB hypophosphorylation and permanent blockage of S phase entry. Importantly, $\mathrm{p} 16^{\mathrm{INK} 4 \mathrm{~A}}$ is a biomarker of natural aging, as its expression increases in a variety of tissues with time (28). Furthermore, studies using a rapidly aging mouse model with deficiencies in the mitotic checkpoint protein BUBR1 (hereafter BUBR1 mice) have demonstrated that prematurely aged adipose tissue and eye and skeletal muscle accumulate p16 ${ }^{\mathrm{INK} 4 \mathrm{~A}}$-expressing senescent cells (20). From a mechanistic perspective, BUBR1 mice, which express about 10\% to $15 \%$ of normal BUBR1, develop progressive aneuploidy, a state of nondiploid numbers of whole chromosomes. These mice are unique, as no other mitotic checkpoint-defective animal exhibits a similar premature aging phenotype, indicating that aneuploidy alone is not sufficient to drive senescence. The underlying molecular alterations driven by BUBR1 insufficiency that promote $\mathrm{p} 16^{\mathrm{INK} 4 \mathrm{~A}}$ expression continue to be the focus of active investigation. Interestingly, genetic inactivation of $\mathrm{p} 16^{\text {INK4A }}$ in BUBR1 mice improved these age-associated features, demonstrating a causal relationship between senescent cell accumulation and tissue deterioration. To further test this theory, approaches to remove senescent cells after they arise have been developed. One such model, termed INK-ATTAC, selectively expresses a drug-inducible FKBP-caspase-8 fusion molecule under 


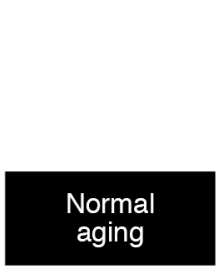

Alzheimer's disease

Parkinson's disease

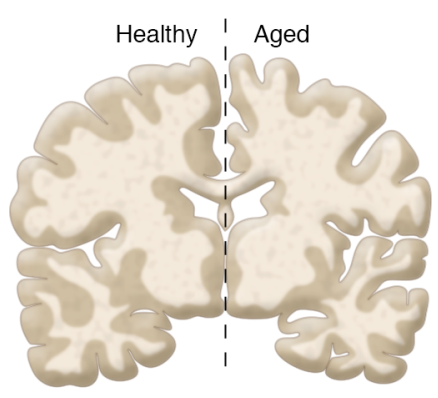

Declining cognition Deficits in memory and processing speed Circadian rhythm/ sleep disruption

Severe cognitive impairment $A \beta$ accumulation Neurofibrillary tangle deposition Microglial activation Brain atrophy
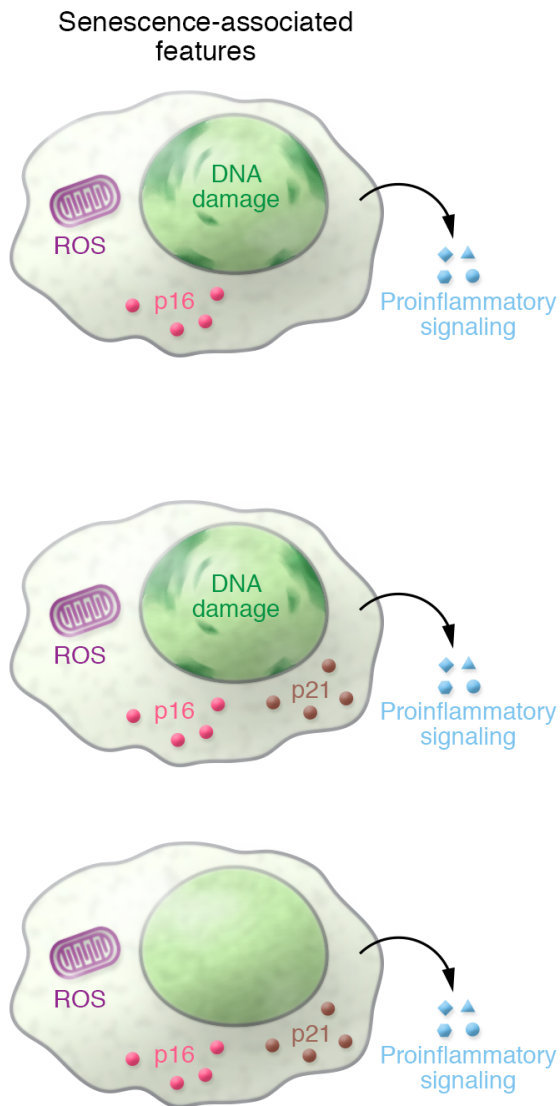

Figure 2. Senescence in normal aging and CNS disease. The molecular correlates of normal aging and CNS disease are not fully defined. Further research on the markers of senescence that distinguish healthy aging from neurodegenerative conditions such as Alzheimer's and Parkinson's disease may provide insights into the role of senescent cells in disease pathogenesis.

the control of a senescent cell-specific portion of the p16 $16^{\text {INK4A }}$ promoter (encompassing $2.6 \mathrm{~kb}$ ) that initiates apoptosis by dimerizing and activating the caspase- 8 fusion proteins when AP20187 is administered to mice (29). Selective elimination of senescent cells in progeroid BUBR1 mice recapitulated the results from the $16^{\mathrm{INK} 4 \mathrm{~A}}$ knockout studies, in which cataracts, sarcopenia, and lipodystrophy were blunted (29). Subsequent studies using the INK-ATTAC model determined that senescent cells drive various natural age-related pathologies, shorten life, and promote the age-associated diseases atherosclerosis and osteoarthritis (20, 29-31).

The observation that senescent cells displayed $\beta$-galactosidase activity at $\mathrm{pH} 6.0$ was an important discovery that helped to establish that senescent cells accumulate with age and at sites of age-related pathology in vivo (32). This activity, now referred to as senescence-associated $\beta$-galactosidase (SA $\beta$-gal), is routinely used in a simple colorimetric assay to detect senescent cells (Figure 1). Another distinguishing feature of senescence is the expression of the SASP, which consists of a variety proteases, growth factors, and inflammatory cytokines $(9,33)$. The upregulation of these proinflammatory molecules has led to a plausible link between senescent cells and "inflammaging" $(34,35)$, an idea that chronic inflammation contributes to age-related disease and tissue dysfunction.

In response to a variety of stimuli, including oncogene activation or loss of certain tumor suppressors, cells can undergo either senescence or apoptosis as a means to prevent neoplastic transfor- mation. A key molecular determinant of this decision is the degree to which $\mathrm{p} 53$ is activated. Excessive signaling through the p 53 pathway triggers cell death through an upregulation of proapoptotic modulators including NOXA and PUMA. Milder "senescence-inducing" stresses promote p53-mediated transcription of the CDKi p21 and the antiapoptotic BCL-2 family members, including BCL-2, BCL-W, and BCL-XL (36). These proteins sequester BH3 domain-containing, proapoptotic proteins to inhibit mitochondrial outer membrane permeabilization and apoptosis (37). Transient low-grade p53 activation facilitates repair; however, prolonged activation can drive cells into senescence. These conditions induce antiapoptotic proteins to promote survival, which may explain why senescent cells accumulate in vitro with passaging and with advancing age in vivo.

Additional features observed in senescent cells include prolonged DNA damage signaling (38), nuclear loss of lamin B1 (39) or HMGB1 (40), and heterochromatic foci formation, although these may not occur in all forms of senescence (Figure 1). It is important to note that while these changes are indicative of senescence, there is currently no single marker that can be used independently to define a senescent cell. These cellular changes, including SA $\beta$-gal, can occur outside of the context of senescence, which necessitates the use of multiple hallmarks of senescence.

An area of intense debate within the senescence field is whether cells that have undergone a terminal differentiation into a nonproliferating cell (e.g., neurons) are capable of acquir- 
ing additional features of senescence besides being permanently growth-arrested. Studies have demonstrated that neurons of aged mice accumulate high amounts of DNA damage (16) and display elevated expression of proinflammatory molecules and SA $\beta$-gal activity (41), characteristics typical of senescence. However, whether these neurons are performing their intended cellular function appropriately (i.e., these features are simply indicators of cellular aging) or are dysfunctional and are negatively influencing other cells in the neighboring environment through the SASP has not been shown experimentally. With the advent of senescent cell-targeting mouse models and therapies (known as senolytics, which are discussed later), studies directed at answering these questions are possible.

\section{Evidence for senescence in the CNS}

The brain requires multiple cell types, including neurons, astrocytes, microglia, oligodendrocytes, and endothelial cells, working in concert to perform activities of day-to-day life. Just like in peripheral tissues, aging leads to a chronic low-grade inflammation in the brain (42), which occurs in the absence of any obvious pathogen. This contributes to decreased pre- and postsynaptic densities, as well as decreased synapses and dendritic spines overall (43), resulting in cognitive impairments and memory loss in aged individuals. Additionally, excessive inflammation, above and beyond chronic low-grade levels accompanying aging, is a common hallmark observed in many neurodegenerative diseases, suggesting that both normal aging and pathological alterations converge on common proinflammatory pathways. The question then arises, what cells might be producing these signals in the brain, and is there any evidence to suggest they have become senescent?

Neurons. As alluded to above, terminally differentiated neurons may exhibit certain features of senescence with advancing age in vivo. Telomere attrition is inherently linked to proliferation; therefore this process is unlikely to occur in postmitotic cells, such as neurons and oligodendrocytes, under normal circumstances. Interestingly, in the context of neurodegenerative diseases, leukocytes of AD patients exhibit shorter telomeres (44), whereas those of PD patients do not (45). It will be interesting to determine whether this feature is reflected in brain tissue from these same patient groups. Furthermore, as no single marker is capable of delineating a senescent cell, it will be imperative to separate out the features that specifically indicate senescence in neurons as opposed to reflecting cellular aging. Perhaps aged neurons are less functional than their youthful counterparts, but, importantly, they have not transitioned into a novel senescent phenotype. Studies aimed at identifying cell types that have lost replicative capacity through differentiation processes are clearly warranted and are lacking in the literature.

Microglia. Microglia, which originate in the bone marrow during development (46), are the primary immune cells of the brain and are functionally analogous to macrophages. In response to invading pathogens or injury, normally quiescent microglia become activated and secrete various growth factors and cytokines and stimulate phagocytosis (47). Microglia from aged mice exhibit increased production of the proinflammatory cytokines IL-6, IL-1 $\beta$, and TNF- $\alpha(48,49)$, all of which are factors upregulated in senescent cells (9). Furthermore, naturally aged micro- glia exhibit shorter telomeres (50). In vitro, chronically activated microglia exhibit various features of senescence, including SA $\beta$-gal activity, heterochromatic foci formation, and growth arrest (51). Future studies to delineate the molecular alterations that define normally activated and senescent microglia will advance the understanding of how these differences may impact age and disease of the CNS.

Astrocytes. Astrocytes, one of the most abundant cell types in the brain, were originally proposed to be nonfunctional fillers of the neuronal network. However, with time and advances, the importance of these cells for many diverse biological processes has been elucidated. Astrocytes are important for maintaining homeostasis through osmotic balance (52), providing metabolic support to neurons (53), and establishing and maintaining the blood-brain barrier (BBB) (54). Through their proximity to neurons, they participate in the tripartite synapse, which couples neurotransmission between pre- and postsynaptic elements (55). There, astrocytes facilitate transmission between neurons and participate in neurotransmitter recycling. Furthermore, astrocytes contribute to defense against trauma, infection, and neurodegeneration to maintain neuronal health and function.

Numerous studies have investigated whether aged astrocytes display features reminiscent of senescence (56). With age, human brain tissue displays an increase in the number of astrocytes positive for $\mathrm{p} 16^{\mathrm{INK} 4 \mathrm{~A}}$ and matrix metalloproteinase 3 (MMP3) (57), a common SASP-associated protease. Astrocytes undergo senescence in vitro in response to ROS exposure, characterized by the presence of SA $\beta$-gal activity, growth arrest, and increased expression of both p16 ${ }^{\mathrm{INK} 4 \mathrm{~A}}$ and p21 (58). Exposure to ionizing radiation induces excessive DNA damage that is also capable of inducing senescence and SASP production in human astrocytes (59). Perhaps not surprisingly, additional studies on the transcriptional alterations accompanying ROS-induced senescence in human astrocytes indicate that genes associated with neuronal development and differentiation are downregulated and proinflammatory genes are upregulated (60). Interestingly, senescent astrocytes also downregulate genes associated with astrocyte activation. Astrocytes become activated (reactive) as a result of many pathologies, including neurodegenerative diseases (61). If senescence has affected these astrocytes, perhaps they would be unable to mount an effective and normal response to these pathological conditions. Just like microglia, activated astrocytes produce proinflammatory cytokines that overlap with known SASP factors. No study has directly compared activated with senescent astrocytes (or microglia) to determine what specific alterations accompany senescence; however, based on the study by Crowe et al. (60), one would anticipate that these differences do in fact exist.

Other cell types. Whether senescence occurs in other cell types of the aging brain, including endothelial cells, oligodendrocytes, and neural stem cells, is currently unknown (Figure 2). Conceptually, senescence in these fractions could cause disruption of the BBB, impaired myelination, and reduced neurogenesis, respectively. In accordance with senescence impairing adult neurogenesis, genetic inactivation of $\mathrm{p} 16^{\mathrm{INK} 4 \mathrm{~A}}$ in mice results in an increased capacity for self-renewal of neuronal stem cells in the subventricular zone of the olfactory bulb of aged mice (62). 
In addition to better mechanistic characterization of the senescent alterations in astrocytes and microglia, it will be important to determine how all of these cells contribute to aging and disease of the CNS.

Interestingly, the prematurely aged BUBR1 mutant mouse displays alterations in the CNS that are typically associated with advanced age. For example, these mice exhibit rampant cerebral gliosis, consisting of activated astrocytes and microglia, very early in life (63). Another feature of normal aging is disruption of the $\mathrm{BBB}$, which leads to loss of homeostasis and aberrant entry of circulating cells and molecules $(64,65)$. BUBR1 hypomorphic mice display leakage across the $\mathrm{BBB}$ that correlates with a propensity for neural endothelial cells and pericytes to prematurely senesce, become SA $\beta$-gal positive, and express p16 $6^{\mathrm{INK} 4 \mathrm{~A}}(66)$. Furthermore, BUBR1 insufficiency impairs adult neurogenesis (67) and oligodendrocyte progenitor cell proliferation in vivo (68), two hallmarks known to decline with natural aging. It is interesting to speculate that premature senescence may underlie all of these pathological alterations in BUBR1 hypomorphic mice. Studies in which senescent cells are eliminated from BUBR1 progeroid mice using the INK-ATTAC transgene are under way to determine whether these cells are to blame for these various pathologies.

\section{Evidence for senescence in CNS aging and disease}

Normal aging is associated with declines in cognition, including deficits in memory and processing speed. As indicators of senescence are expressed in a variety of cell types of the CNS with age, it is tempting to speculate that senescent cells negatively influence cognition. However, no direct links have yet been established for a relationship during normative aging. A number of human progeria syndromes, including Werner syndrome, Cockayne syndrome, ataxia telangiectasia, and Down syndrome (69), exhibit premature senescence. Interestingly, patients with certain progerias, including Down syndrome (70), ataxia telangiectasia (71), and Cockayne syndrome (72), demonstrate cognitive deficits, whereas Werner syndrome and Hutchinson-Gilford (73) patients do not. It would be informative to determine whether senescent cells are found in the CNS of patients with these various progerias and to identify the cells' lineages, as they may point to molecular entry points for investigation in the normal aging process.

Unlike human studies, many of the experiments used to test memory and cognition in mouse models rely on behavioral alterations in response to stimuli, including visual cues and adverse stimuli like water. Testing BUBR1 mice with these approaches is complicated by the fact that they develop severe skeletal muscle atrophy and cataracts early in life, so their cognitive ability is largely unknown. The senescence-accelerated mouse (SAMP8) is a prematurely aged mouse model that developed spontaneously from phenotypic selection of mice (74). In addition to a shortened lifespan, SAMP8 mice develop a number of premature age-related phenotypes, including impaired learning and memory (reviewed in ref. 75). These deficits are linked to overproduction of amyloid precursor protein and amyloid- $\beta$ (A $\beta$ ) (76), which are known to contribute to neurodegenerative disease in people.

Alzheimer's disease. $\mathrm{AD}$, the most prevalent neurodegenerative disease in humans, is characterized by the neuropathological accumulation of $A \beta$ peptide-containing amyloid plaques and neurofibrillary tangle aggregates of hyperphosphorylated or misfolded tau protein (77). These alterations negatively impact neurons, thereby leading to cognitive impairment and neurodegeneration. The cell types prone to develop features of senescence during normal aging also seem to demonstrate alterations in $\mathrm{AD}$. For example, microglia from $\mathrm{AD}$ patients possess shorter telomeres compared with age-matched controls (50). Chronic microglial activation, which is commonly observed in $\mathrm{AD}$, mounts an acute immune response against misfolded proteins like $A \beta$ that may actively drive neuronal death through excessive neurotoxic factor production (78). Astrocytes cultured from patients with $\mathrm{AD}$ express not only more of the CDKi p $16^{\text {INK4A }}$ than age-matched controls, but also more MMP1 and IL-6 (57). Elevated markers of DNA damage, which are also a distinguishing characteristic of senescent cells (38), occur in the astrocytes of dementia patients (79). Interestingly, neurons with neurofibrillary tangles have increased expression of $\mathrm{p} 16^{\mathrm{INK} 4 \mathrm{~A}}(80-82)$; however, it is very unclear whether this induction occurs in the context of true senescence. A true causal relationship between senescent cell accumulation and AD has not been established, indicating that further work is needed in this area. While continuous progress is being made on understanding the biology behind $\mathrm{AD}$, including where and when amyloid plaques are deposited during disease progression and how to remove them, clinical trials of amyloid-centered therapeutics have had disappointing outcomes (83). Novel approaches are clearly needed and could include trials to target tau or senescent cells, if they are found to drive AD.

Parkinson's disease. Patients with PD, the second most common neurodegenerative disease, display characteristic loss of motor control due to loss of dopamine-producing neurons in the substantia nigra. Although it is associated with increasing age, its pathogenesis is not well understood. Environmental exposure and genetic risk factors that predispose people to PD development represent only a minor fraction of the cases, suggesting that the etiology is inherently more complex. Interestingly, a common feature of PD patients and mouse models is the accumulation of activated microglia and astrocytes, which would promote increased local concentrations of inflammatory mediators and ROS (84). This prolonged chronic inflammatory state may therefore shift microglial function toward neurodegeneration (85). Whether these microglia or astrocytes display features distinctive of senescence remains to be established.

Other neurodegenerative conditions. Researchers have begun to explore whether senescent cells are found in additional neurodegenerative pathologies. For example, HIV patients are prone to develop a number of complications indicative of premature aging, including effects on neurological function collectively referred to as HIV-associated neurocognitive disorders (HAND) (86). HAND can range in severity from asymptomatic to demented. It has been postulated that the antiretroviral drugs given to HIV patients to manage disease in fact promote HAND severity. Indeed, treating primary astrocytes in vitro with these drugs promotes senescence $(87,88)$. Whether microglia develop characteristics associated with senescence after similar exposures has yet to be determined (89). These recent works demonstrate that our understanding of the contribution of senescent cells to neurodegenerative processes is likely in its infancy. 


\section{Senescent cells - a therapeutic opportunity for neurodegenerative disease?}

The above studies suggest that senescent cells may accumulate with age and at sites of pathology in the brain. However, the significance of senescent cells in both the induction and exacerbation of neurodegenerative disorders is unknown. To date, it has been extremely difficult to discern the overall significance and mechanistic contribution of senescent cells to neuropathology because of a lack of tools to identify, isolate, and/or eliminate these cells. Current methods rely on a combination of markers, including those described in Figure 1. As these markers are not truly unique to senescent cells, it is imperative to establish that these changes occur in the context of senescence and not just as a result of inflammation, for example. A true demonstration that senescent cells causally drive neuropathology can be achieved either by prevention of senescence entry through genetic inactivation of $\mathrm{p} 16^{\mathrm{INK} 4 \mathrm{~A}}$ or by direct elimination of senescent cells using genetically engineered mice. To date, these approaches have not been employed for relevant studies of neurodegenerative disease. One limitation of genetic inactivation experiments is that mice lacking $\mathrm{p} 16^{\mathrm{INK} 4 \mathrm{~A}}$ are highly tumor prone and therefore die before the cognitive decline is observed in normal mice (90). To circumvent this problem, two similar yet unique mouse models have been developed to detect and eliminate senescent cells. The INK-ATTAC transgenic mouse (described above) employs a $2.6-\mathrm{kb}$ promoter element of $\mathrm{p} 16^{\mathrm{INK} 4 \mathrm{~A}}$ to drive expression of drugresponsive caspase-8 (29). A second transgenic model, termed p16-3MR, expresses a trimodal reporter construct under the control of a bacterial artificial chromosome containing the entire p16 ${ }^{\text {INK4A }}$ promoter (91). This reporter contains monomeric red fluorescent protein (mRFP), synthetic Renilla luciferase, and a truncated herpes simplex virus thymidine kinase (HSV-TK). When ganciclovir is administered, HSV-TK converts this compound into a toxic DNA chain terminator in $\mathrm{p} 16^{\mathrm{INK} 4 \mathrm{~A}}$-expressing cells, triggering apoptosis in senescent cells through mitochondria-dependent cell death (92). Use of these models has dramatically accelerated our understanding of the contribution of senescent cells to various age-associated alterations and pathologies, including osteoarthritis and atherosclerosis $(30,31)$.

The finding that senescent cell elimination is not only possible but also effective at modulating inflammatory diseases has triggered profound interest in developing therapeutic strategies to eliminate senescent cells in the absence of genetic modifications. These "senolytic" approaches eliminate not only the senescent cell but also the accompanying SASP, which likely exerts beneficial effects in the local environment where senescent cells reside. Several senolytic molecules have already been identified. Many of these agents target the antiapoptotic machinery that is upregulated in senescent cells. The senolytic molecules ABT-737 (37) and navitoclax (ABT-263) (93) occupy the inhibitory binding regions of members of the BCL-2 family of proteins, thereby canceling their antiapoptotic activity. Navitoclax treatment has been shown to eliminate senescent cells that arise in early atherosclerotic plaques (30) and upon sublethal irradiation in both skeletal muscle and the bone marrow (93). In atherosclerosis, senescent cells contribute atheroma initiation and maturation, and navitoclax administration reduces plaque burden (30). Other potentially senolytic molecules and strategies, including piperlongumine (94), dasatinib (95), quercetin (95), the proprietary compound UBX0101 (31), a FOXO4 peptide that promotes p53-dependent apoptosis (96), and HSP9O inhibitors (97), have also been identified, demonstrating that there are many possible treatment options for senescent cell removal in aging and diseases. Interestingly, these various studies demonstrate that whatever means is employed, targeting of senescent cells has largely beneficial impacts for pathologies of aging and age-related disease. Therefore, if these approaches are to be implemented in neurological conditions, there are likely a number of candidates that demonstrate bioavailability in the brain, in the context of both aging and disease. The BBB may preclude systemic administration for some of these compounds to get to the target site.

At first, it will be extremely difficult to justify the use of senolytic molecules outside the context of a clear disease as a prevention strategy to delay various diseases associated with senescent cell accumulation. Treatments to extend function in otherwise normal individuals will follow after demonstration that interventions targeting senescent cells have beneficial impacts in pathological conditions. Extensive preclinical testing will need to be performed in order to identify the best senolytic candidate for neurodegenerative diseases. The best candidate disease model for initial trials will have demonstrated a link between senescent cell accumulation and disease. Once identified, it is imperative to demonstrate that senescent cells are causally implicated in the disease. The senolytic therapy will then need to demonstrate senescent cell ablation in the context of disease. The expectation would then be that senolytic therapy reduces the SASP and markers of disease. Subsequent clinical trials will be needed to determine how these treatments improve diseases that accumulate senescent cells locally. These disease-modulating impacts are far more critical, as impacts on overall health and lifespan of people are outside the scope of most clinical trials because of the length of time required to observe such effects.

In order to bring a potential senolytic therapy into the clinic, a number of important challenges need to be considered. Several important insights into the effectiveness of senescent cell elimination strategies have used tissue culture conditions or mouse models. It is currently unclear how these various senolytics can be translated into clinical strategies, if at all. Furthermore, the duration of treatments has been brief with relatively short follow-up assessments, so long-term negative consequences have not been observed in studies to date. In the study using naturally aged mice, senescent cell elimination with the INK-ATTAC transgenic construct had no discernible negative side effects (98). It is important to keep in mind that the animals used in this study were in a controlled environment without any exogenous stresses applied. In response to certain conditions, such as wounding, the inability to acutely induce senescent cells results in a retarded rate of wound closure $(91,98)$. The aforementioned studies demonstrate a beneficial role for senescence in tissue repair and remodeling in the short term, whereas the long-term consequences appear to be largely detrimental. Proof that senescent cells contribute to pathologies and aging of the CNS is imperative to provide the foundation on which future strategies will build. 


\section{Outlook}

Organismal aging and age-related diseases promote an accumulation of senescent cells in a variety of tissues in both humans and mice (8). Using mouse models, elimination of these cells, through either genetic means or senolytic therapies, has largely shown beneficial impacts, irrespective of the disease or condition investigated $(29-31,99,100)$. With these tools, we are now positioned to critically test the concept that senescent cells promote age-related diseases and pathology in the CNS. Limited and indirect evidence suggests a potential accumulation of these cells with age and dysfunction. With the advent of novel mouse models and approaches for senescent cell elimination with senolytics, we can establish when senescent cells arise during the disease process and how they contribute to these alterations. Alternatively, to assess the disease-initiating contribution of senescent cells, techniques to induce senescence, such as irradiation or chemotherapeutics, can be used to increase the burden to study how this impacts the rate of pathology. If senescent cells are indeed active drivers of neurodegeneration and not simply bystanders in the process, this could lead to the development of long-awaited therapeutics to ameliorate these devastating diseases or at least increase the years of healthy life free from dysfunction. This is especially important, as many clinical trials to combat neurodegeneration using previously established molecular targets have consistently failed, leading to a sense of desperation. A major criticism of these trials is that treatment was started too late in the disease process to have beneficial impacts. Perhaps once neurodegenerative processes are initiated, there is a point of no return.

Diversifying and exploring new treatment ideas is essential, as these diseases are inherently complex and not terribly well understood. In order to be maximally effective, clinical trials will need to recruit the right patients, predict those at highest risk for disease development, and begin treatment at the right age. The determination of populations of high risk is yet another area in the field that requires significantly more research, as there is currently no reliable way to identify the early signs of these devastating diseases. Once these highest-risk populations are determined, interventions can be started prior to neuronal loss, which likely would result in the highest chance for success.

\section{Acknowledgments}

The authors thank Tyler Bussian, Charlie Meyer, and Bennett Childs for helpful feedback on the manuscript. This work was supported by the NIH (R01AG053229 to DJB, P50AG016574 to RCP, and U01AG006786 to RCP), the Glenn Foundation for Medical Research (to DJB), the Ellison Medical Foundation (to DJB), the Mayo Clinic Children's Research Center (to DJB), and the Alzheimer's Disease Research Center of Mayo Clinic (to RCP and DJB).

Address correspondence to: Darren J. Baker, Mayo Clinic, 200 First Street SW, Rochester, Minnesota 55905, USA. Phone: 507.538.4097; Email: baker.darren@mayo.edu.
1. Hebert LE, Weuve J, Scherr PA, Evans DA. Alzheimer disease in the United States (2010-2050) estimated using the 2010 census. Neurology. 2013;80(19):1778-1783.

2. Kukull WA, et al. Dementia and Alzheimer disease incidence: a prospective cohort study. Arch Neurol. 2002;59(11):1737-1746.

3. Hurd MD, Martorell P, Delavande A, Mullen KJ, Langa KM. Monetary costs of dementia in the United States. NEngl JMed. 2013;368(14):1326-1334.

4. Campisi J, Robert L. Cell senescence: role in aging and age-related diseases. Interdiscip Top Gerontol. 2014;39:45-61.

5. Jeyapalan JC, Sedivy JM. Cellular senescence and organismal aging. Mech Ageing Dev. 2008; 129(7-8):467-474.

6. Sharpless NE, Sherr CJ. Forging a signature of in vivo senescence. Nat Rev Cancer. 2015;15(7):397-408.

7. van Deursen JM. The role of senescent cells in ageing. Nature. 2014;509(7501):439-446.

8. Childs BG, et al. Senescent cells: an emerging target for diseases of ageing. Nat Rev Drug Discov. 2017;16(10):718-735.

9. Coppé JP, et al. Senescence-associated secretory phenotypes reveal cell-nonautonomous functions of oncogenic RAS and the p53 tumor suppressor. PLoS Biol. 2008;6(12):2853-2868.

10. Hayflick L, Moorhead PS. The serial cultivation of human diploid cell strains. Exp Cell Res. 1961;25:585-621.

11. Hayflick L. The limited in vitro lifetime of human diploid cell strains. Exp Cell Res. 1965;37:614-636.

12. Bodnar AG, et al. Extension of life-span by introduction of telomerase into normal human cells. Science. 1998;279(5349):349-352.
13. Olovnikov AM. A theory of marginotomy. The incomplete copying of template margin in enzymic synthesis of polynucleotides and biological significance of the phenomenon. J Theor Biol. 1973;41(1):181-190.

14. Blasco MA, et al. Telomere shortening and tumor formation by mouse cells lacking telomerase RNA. Cell. 1997;91(1):25-34.

15. Muñoz-Espín D, Serrano M. Cellular senescence: from physiology to pathology. Nat Rev Mol Cell Biol. 2014;15(7):482-496.

16. Sedelnikova OA, Horikawa I, Zimonjic DB, Popescu NC, Bonner WM, Barrett JC. Senescing human cells and ageing mice accumulate DNA lesions with unrepairable double-strand breaks. Nat Cell Biol. 2004;6(2):168-170.

17. von Zglinicki T. Oxidative stress shortens telomeres. Trends Biochem Sci. 2002;27(7):339-344.

18. Di Micco R, et al. Oncogene-induced senescence is a DNA damage response triggered by DNA hyper-replication. Nature. 2006;444(7119):638-642.

19. Alimonti A, et al. A novel type of cellular senescence that can be enhanced in mouse models and human tumor xenografts to suppress prostate tumorigenesis. JClin Invest. 2010;120(3):681-693.

20. Baker DJ, et al. Opposing roles for p16In$\mathrm{k} 4 \mathrm{a}$ and p19Arf in senescence and ageing caused by BubR1 insufficiency. Nat Cell Biol. 2008;10(7):825-836.

21. Bartkova J, et al. Oncogene-induced senescence is part of the tumorigenesis barrier imposed by DNA damage checkpoints. Nature. 2006;444(7119):633-637.

22. Romanov VS, et al. p21(Waf1) is required for cellular senescence but not for cell cycle arrest induced by the HDAC inhibitor sodium butyrate. Cell Cycle. 2010;9(19):3945-3955.

23. Eischen CM, Lozano G. The Mdm network and its regulation of $\mathrm{p} 53$ activities: a rheostat of cancer risk. Hum Mutat. 2014;35(6):728-737.

24. Shiloh Y, Ziv Y. The ATM protein kinase: regulating the cellular response to genotoxic stress, and more. Nat Rev Mol Cell Biol. 2013;14(4):197-210.

25. Herbig U, Wei W, Dutriaux A, Jobling WA, Sedivy JM. Real-time imaging of transcriptional activation in live cells reveals rapid up-regulation of the cyclin-dependent kinase inhibitor gene CDKN1A in replicative cellular senescence. Aging Cell. 2003;2(6):295-304.

26. Wong ES, et al. p38MAPK controls expression of multiple cell cycle inhibitors and islet proliferation with advancing age. Dev Cell. 2009;17(1):142-149.

27. Ohtani N, et al. Opposing effects of Ets and Id proteins on p16INK4a expression during cellular senescence. Nature. 2001;409(6823):1067-1070.

28. Krishnamurthy J, et al. Ink4a/Arf expression is a biomarker of aging. J Clin Invest. 2004;114(9):1299-1307.

29. Baker DJ, et al. Clearance of p16Ink4a-positive senescent cells delays ageing-associated disorders. Nature. 2011;479(7372):232-236.

30. Childs BG, Baker DJ, Wijshake T, Conover CA, Campisi J, van Deursen JM. Senescent intimal foam cells are deleterious at all stages of atherosclerosis. Science. 2016;354(6311):472-477.

31. Jeon $\mathrm{OH}$, et al. Local clearance of senescent cells attenuates the development of post-traumatic osteoarthritis and creates a pro-regenerative environment. Nat Med. 2017;23(6):775-781. 
32. Dimri GP, et al. A biomarker that identifies senescent human cells in culture and in aging skin in vivo. Proc Natl Acad Sci U SA.1995;92(20):9363-9367.

33. Coppé JP, et al. A human-like senescence-associated secretory phenotype is conserved in mouse cells dependent on physiological oxygen. PLoS One. 2010;5(2):e9188.

34. Campisi J, d'Adda di Fagagna F. Cellular senescence: when bad things happen to good cells. Nat Rev Mol Cell Biol. 2007;8(9):729-740.

35. Kuilman T, Peeper DS. Senescence-messaging secretome: SMS-ing cellular stress. Nat Rev Cancer. 2009;9(2):81-94.

36. Tavana O, et al. Absence of p53-dependent apoptosis leads to UV radiation hypersensitivity, enhanced immunosuppression and cellular senescence. Cell Cycle. 2010;9(16):3328-3336.

37. Yosef $\mathrm{R}$, et al. Directed elimination of senescent cells by inhibition of BCL-W and BCL-XL. Nat Commun. 2016;7:11190.

38. Rodier F, et al. DNA-SCARS: distinct nuclear structures that sustain damage-induced senescence growth arrest and inflammatory cytokine secretion. JCell Sci. 2011;124(pt 1):68-81.

39. Freund A, Laberge RM, Demaria M, Campisi J. Lamin B1 loss is a senescence-associated biomarker. Mol Biol Cell. 2012;23(11):2066-2075.

40. Davalos AR, et al. p53-dependent release of Alarmin HMGB1 is a central mediator of senescent phenotypes. JCell Biol. 2013;201(4):613-629.

41. Jurk D, et al. Postmitotic neurons develop a p21-dependent senescence-like phenotype driven by a DNA damage response. Aging Cell. 2012;11(6):996-1004.

42. Frank-Cannon TC, Alto LT, McAlpine FE, Tansey MG. Does neuroinflammation fan the flame in neurodegenerative diseases? Mol Neurodegener. 2009;4:47.

43. Yankner BA, Lu T, Loerch P. The aging brain. Annu Rev Pathol. 2008;3:41-66.

44. Forero DA, González-Giraldo Y, López-Quintero C, Castro-Vega LJ, Barreto GE, Perry G. Meta-analysis of telomere length in Alzheimer's disease. J Gerontol A Biol Sci Med Sci. 2016;71(8):1069-1073.

45. Forero DA, González-Giraldo Y, López-Quintero C, Castro-Vega LJ, Barreto GE, Perry G. Telomere length in Parkinson's disease: a meta-analysis. Exp Gerontol. 2016;75:53-55.

46. Ginhoux F, et al. Fate mapping analysis reveals that adult microglia derive from primitive macrophages. Science. 2010;330(6005):841-845.

47. Doorn KJ, et al. Emerging roles of microglial activation and non-motor symptoms in Parkinson's disease. Prog Neurobiol. 2012;98(2):222-238.

48. Sierra A, Gottfried-Blackmore AC, McEwen BS, Bulloch K. Microglia derived from aging mice exhibit an altered inflammatory profile. Glia. 2007;55(4):412-424.

49. Bachstetter AD, Xing B, de Almeida L, Dimayuga ER, Watterson DM, Van Eldik LJ. Microglial p38 $\alpha$ MAPK is a key regulator of proinflammatory cytokine up-regulation induced by toll-like receptor (TLR) ligands or beta-amyloid (A $\beta$ ). J Neuroinflammation. 2011;8:79.

50. Flanary BE, Sammons NW, Nguyen C, Walker D, Streit WJ. Evidence that aging and amyloid promote microglial cell senescence. Rejuvenation
Res. 2007;10(1):61-74.

51. Yu HM, et al. Repeated lipopolysaccharide stimulation induces cellular senescence in BV2 cells. Neuroimmunomodulation. 2012;19(2):131-136.

52. Abbott NJ, Ronnback L, Hansson E. Astrocyte-endothelial interactions at the blood-brain barrier. Nat Rev Neurosci. 2006;7(1):41-53.

53. Newington JT, Harris RA, Cumming RC. Reevaluating metabolism in Alzheimer's disease from the perspective of the astrocyte-neuron lactate shuttle model. J Neurodegener Dis. 2013;2013:234572.

54. Lécuyer MA, Kebir H, Prat A. Glial influences on BBB functions and molecular players in immune cell trafficking. Biochim Biophys Acta. 2016;1862(3):472-482.

55. Araque A, Parpura V, Sanzgiri RP, Haydon PG. Tripartite synapses: glia, the unacknowledged partner. Trends Neurosci. 1999;22(5):208-215.

56. Salminen A, Ojala J, Kaarniranta K, Haapasalo A, Hiltunen M, Soininen $\mathrm{H}$. Astrocytes in the aging brain express characteristics of senescence-associated secretory phenotype. Eur J Neurosci. 2011;34(1):3-11.

57. Bhat $\mathrm{R}$, et al. Astrocyte senescence as a component of Alzheimer's disease. PLoS One. 2012;7(9):e45069.

58. Bitto A, et al. Stress-induced senescence in human and rodent astrocytes. Exp Cell Res. 2010;316(17):2961-2968.

59. Zou Y, et al. Responses of human embryonic stem cells and their differentiated progeny to ionizing radiation. Biochem Biophys Res Commun. 2012;426(1):100-105.

60. Crowe EP, et al. Changes in the transcriptome of human astrocytes accompanying oxidative stress-induced senescence. Front Aging Neurosci. 2016;8:208.

61. Pekny M, Nilsson M. Astrocyte activation and reactive gliosis. Glia. 2005;50(4):427-434.

62. Molofsky AV, et al. Increasing p16INK4a expression decreases forebrain progenitors and neurogenesis during ageing. Nature. 2006;443(7110):448-452.

63. Hartman TK, Wengenack TM, Poduslo JF, van Deursen JM. Mutant mice with small amounts of BubR1 display accelerated age-related gliosis. Neurobiol Aging. 2007;28(6):921-927.

64. Zlokovic BV. Neurovascular pathways to neurodegeneration in Alzheimer's disease and other disorders. Nat Rev Neurosci. 2011;12(12):723-738.

65. Montagne A, et al. Blood-brain barrier breakdown in the aging human hippocampus. Neuron. 2015;85(2):296-302.

66. Yamazaki Y, et al. Vascular cell senescence contributes to blood-brain barrier breakdown. Stroke. 2016;47(4):1068-1077.

67. Yang Z, et al. Age-related decline in BubR1 impairs adult hippocampal neurogenesis. Aging Cell. 2017;16(3):598-601.

68. Choi CI, et al. The progeroid gene BubR1 regulates axon myelination and motor function. Aging (Albany NY). 2016;8(11):2667-2688.

69. Weirich-Schwaiger H, Weirich HG, Gruber B, Schweiger M, Hirsch-Kauffmann M. Correlation between senescence and DNA repair in cells from young and old individuals and in premature aging syndromes. Mutat Res. 1994;316(1):37-48.
70. Silverman W. Down syndrome: cognitive phenotype. Ment Retard Dev Disabil Res Rev. 2007;13(3):228-236.

71. Hoche F, et al. Cognitive phenotype in ataxia-telangiectasia. Pediatr Neurol. 2014;51(3):297-310.

72. Karikkineth AC, Scheibye-Knudsen M, Fivenson E, Croteau DL, Bohr VA. Cockayne syndrome: clinical features, model systems and pathways. Ageing Res Rev. 2017;33:3-17.

73. Nissan $X$, et al. Unique preservation of neural cells in Hutchinson-Gilford progeria syndrome is due to the expression of the neural-specific miR-9 microRNA. Cell Rep. 2012;2(1):1-9.

74. Takeda T, et al. A new murine model of accelerated senescence. Mech Ageing Dev. 1981;17(2):183-194.

75. Morley JE, Armbrecht HJ, Farr SA, Kumar VB. The senescence accelerated mouse (SAMP8) as a model for oxidative stress and Alzheimer's disease. Biochim Biophys Acta. 2012;1822(5):650-656.

76. Morley JE, et al. $\beta$-Amyloid precursor polypeptide in SAMP8 mice affects learning and memory. Peptides. 2000;21(12):1761-1767.

77. Crews L, Masliah E. Molecular mechanisms of neurodegeneration in Alzheimer's disease. Hum Mol Genet. 2010;19(R1):R12-R20.

78. Sugama S. Stress-induced microglial activation may facilitate the progression of neurodegenerative disorders. Med Hypotheses. 2009;73(6):1031-1034.

79. Myung NH, et al. Evidence of DNA damage in Alzheimer disease: phosphorylation of histone H2AX in astrocytes. Age (Dordr). 2008;30(4):209-215.

80. Arendt T, Holzer M, Gärtner U. Neuronal expression of cycline dependent kinase inhibitors of the INK4 family in Alzheimer's disease. J Neural Transm (Vienna). 1998;105(8-9):949-960.

81. Arendt T, Rödel L, Gärtner U, Holzer M. Expression of the cyclin-dependent kinase inhibitor p16 in Alzheimer's disease. Neuroreport. 1996;7(18):3047-3049.

82. McShea A, Harris PL, Webster KR, Wahl AF, Smith MA. Abnormal expression of the cell cycle regulators P16 and CDK4 in Alzheimer's disease. Am J Pathol. 1997;150(6):1933-1939.

83. Mehta D, Jackson R, Paul G, Shi J, Sabbagh M. Why do trials for Alzheimer's disease drugs keep failing? A discontinued drug perspective for 2010-2015. Expert Opin Investig Drugs. 2017;26(6):735-739.

84. Ransohoff RM. How neuroinflammation contributes to neurodegeneration. Science. 2016;353(6301):777-783.

85. Calabrese V, et al. Aging and Parkinson's disease: inflammaging, neuroinflammation and biological remodeling as key factors in pathogenesis. Free Radic Biol Med. 2017;115:80-91.

86. Heaton RK, et al. HIV-associated neurocognitive disorders persist in the era of potent antiretroviral therapy: CHARTER Study. Neurology. 2010;75(23):2087-2096.

87. Cohen J, D’Agostino L, Wilson J, Tuzer F, Torres C. Astrocyte senescence and metabolic changes in response to HIV antiretroviral therapy drugs. Front Aging Neurosci. 2017;9:281.

88. Yu C, Narasipura SD, Richards MH, Hu XT, Yamamoto B, Al-Harthi L. HIV and drug abuse mediate astrocyte senescence in a $\beta$-catenindependent manner leading to neuronal toxicity. 
Aging Cell. 2017;16(5):956-965.

89. Chen NC, Partridge AT, Sell C, Torres C, Martín-García J. Fate of microglia during HIV-1 infection: from activation to senescence? Glia. 2017;65(3):431-446.

90. Sharpless NE, et al. Loss of p16Ink4a with retention of p19Arf predisposes mice to tumorigenesis. Nature. 2001;413(6851):86-91.

91. Demaria $\mathrm{M}$, et al. An essential role for senescent cells in optimal wound healing through secretion of PDGF-AA. Dev Cell. 2014;31(6):722-733.

92. Laberge RM, et al. Mitochondrial DNA damage induces apoptosis in senescent cells. Cell Death
Dis. 2013;4:e727.

93. Chang J, et al. Clearance of senescent cells by ABT263 rejuvenates aged hematopoietic stem cells in mice. Nat Med. 2016;22(1):78-83.

94 . Wang Y, et al. Discovery of piperlongumine as a potential novel lead for the development of senolytic agents. Aging (Albany NY). 2016;8(11):2915-2926.

95. Zhu Y, et al. The Achilles' heel of senescent cells: from transcriptome to senolytic drugs. Aging Cell. 2015;14(4):644-658.

96. Baar MP, et al. Targeted apoptosis of senescent cells restores tissue homeostasis in response to chemo- toxicity and aging. Cell.2017;169(1):132-147.e16.

97. Fuhrmann-Stroissnigg H, et al. Identification of HSP90 inhibitors as a novel class of senolytics. Nat Commun. 2017;8(1):422.

98. Baker DJ, et al. Naturally occurring p16(Ink4a)-positive cells shorten healthy lifespan. Nature. 2016;530(7589):184-189.

99. Schafer MJ, et al. Exercise prevents diet-induced cellular senescence in adipose tissue. Diabetes. 2016;65(6):1606-1615.

100.Schafer MJ, et al. Cellular senescence mediates fibrotic pulmonary disease. Nat Commun. 2017;8:14532. 